\title{
Iridescent Cellulose Nanocrystal Films Modified
}

\section{with Hydroxypropyl Cellulose}

Christopher M. Walters, ${ }^{a}$ Charlotte E. Boott, ${ }^{a}$ Thanh-Dinh Nguyen,${ }^{a}$ Wadood Y. Hamad, ${ }^{b}$ and Mark J. MacLachlan*a,c,d

${ }^{\text {a }}$ Department of Chemistry, University of British Columbia, 2036 Main Mall, Vancouver, BC V6T 1Z1, Canada.

${ }^{\mathrm{b}}$ Transformation and Interfaces Group, Bioproducts Innovation Centre of Excellence, FPInnovations, 2665 East Mall, Vancouver, BC, V6T 1Z4, Canada.

${ }^{c}$ Stewart Blusson Quantum Matter Institute, University of British Columbia, 2355 East Mall, Vancouver, BC V6T 1Z4, Canada

${ }^{d}$ WPI Nano Life Science Institute, Kanazawa University, Kanazawa, 920-1192, Japan 


\section{Experimental setup for the preparation of methacrylate-functionalized CNC composite films}

During the functionalization of the CNC composite films, the films are prone to structural damage due to the stir bar. To prevent this, we used the experimental setup illustrated below, where the stir bar was in the same solvent as the film but was separated by a porous ceramic disc (pore size 1 $\mathrm{mm})$

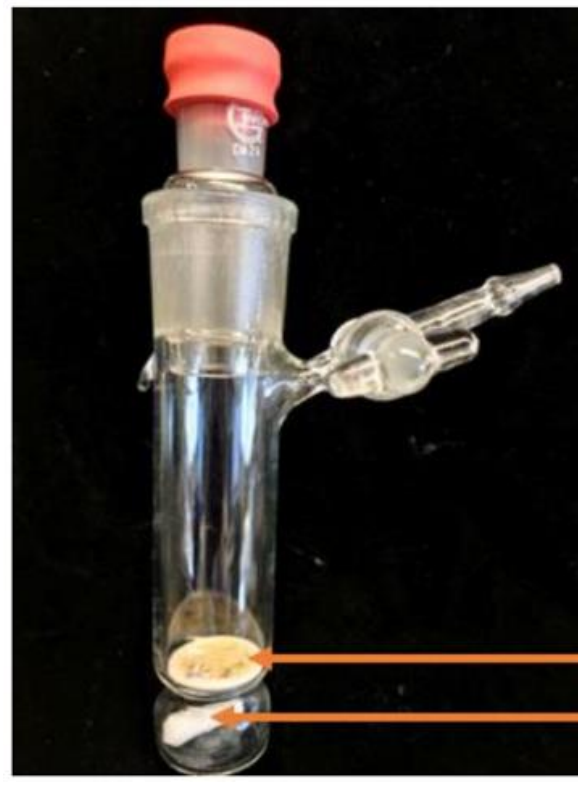

Ceramic disk

Stir Bar

Figure S1. Glassware used to prevent the mechanical breakdown of the films. The ceramic disc forms a barrier between the stir bar and the films yielding an intact film after functionalization.

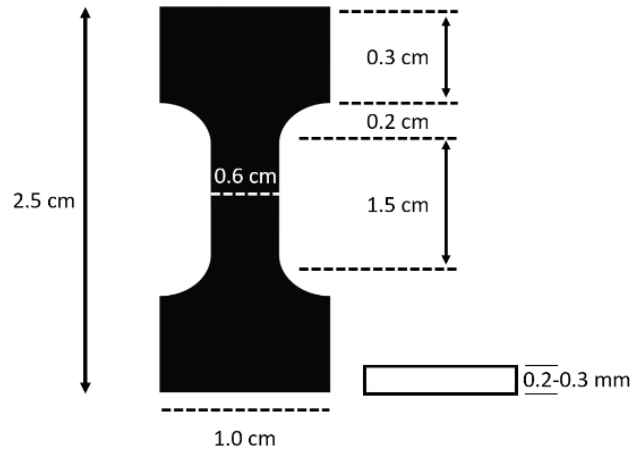

Figure S2. Standard shape of sample used for tensile testing measurements. 

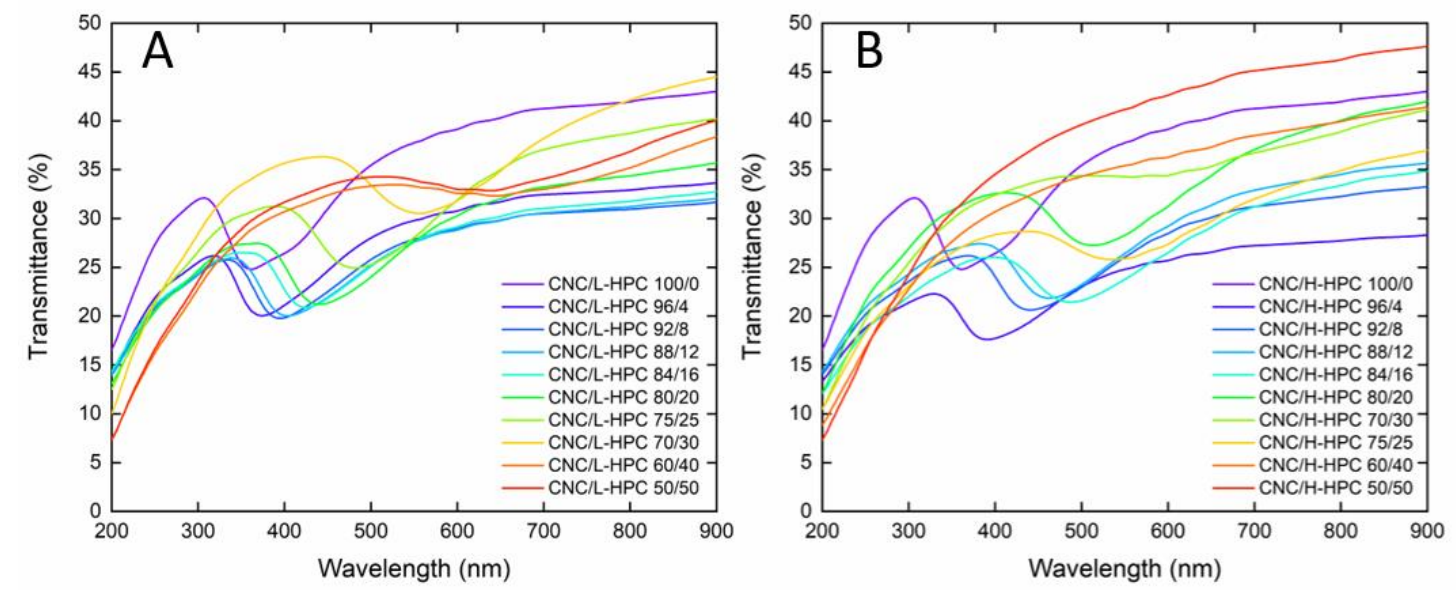

Figure S3. UV-Vis transmittance of A) $\mathrm{CNC} / \mathrm{L}-\mathrm{HPC}$ composites ranging from $50 \mathrm{w} / \mathrm{w} \mathrm{CNC}$ to $100 \mathrm{w} / \mathrm{w}$ CNC. B) CNC/H-HPC composites ranging from $50 \mathrm{w} / \mathrm{w} \mathrm{CNC}$ to $100 \mathrm{w} / \mathrm{w} \mathrm{CNC}$.

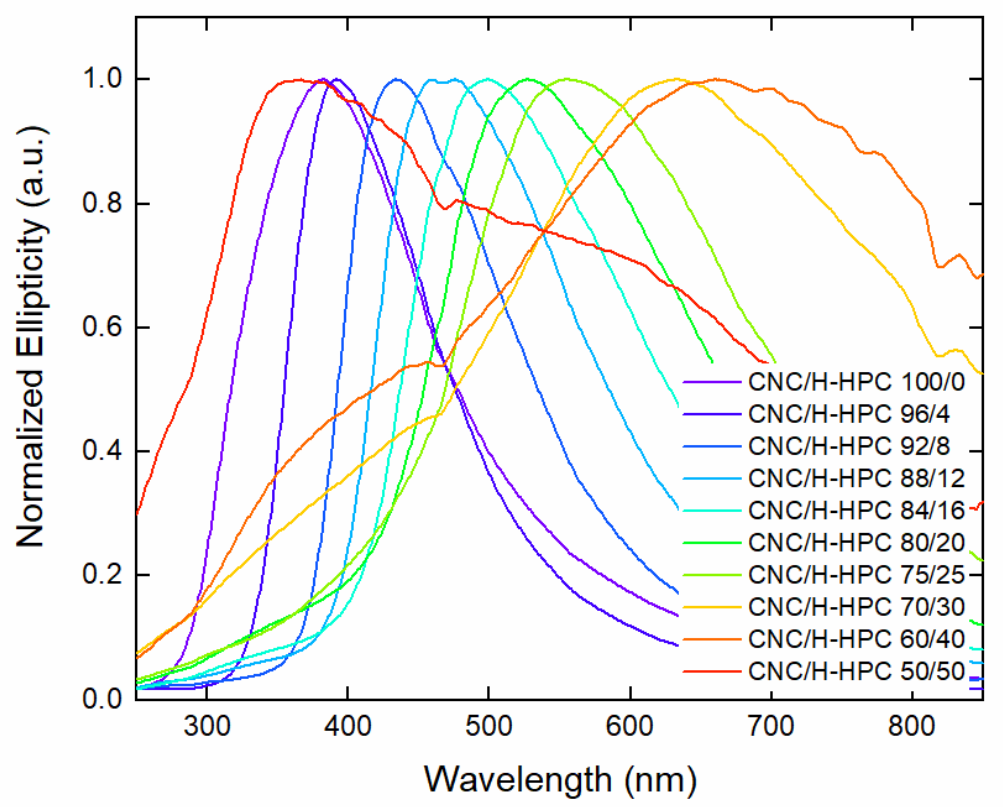

Figure S4. Normalized CD spectra of CNC/H-HPC composites ranging from $50 \mathrm{w} / \mathrm{w}$ CNC to 100 $\mathrm{w} / \mathrm{w} \mathrm{CNC}$. 

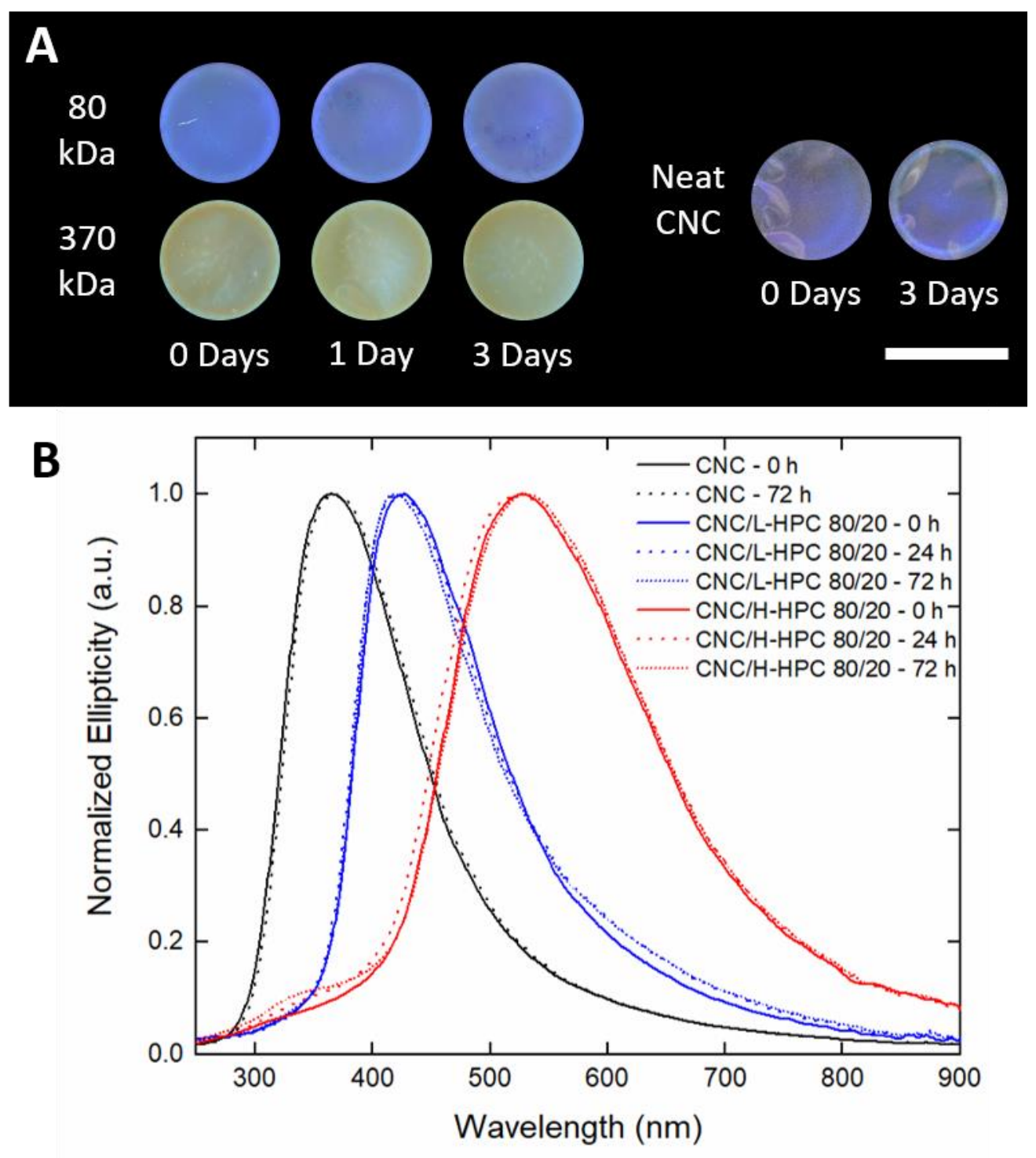

Figure S5. A) Photographs of CNC/HPC composite films where the $\mathrm{CNC}$ and HPC were allowed to equilibrate for 0,1 , and 3 days in a sealed vial prior to evaporation. Films prepared from neat CNCs are also shown at 0 and 3 days. Scale bar is $3.5 \mathrm{~cm}$. B) Normalized CD spectra CNC/HPC composite films where the $\mathrm{CNC}$ and $\mathrm{HPC}$ were allowed to equilibrate for 0,1 , and 3 days in a sealed vial prior to evaporation. 

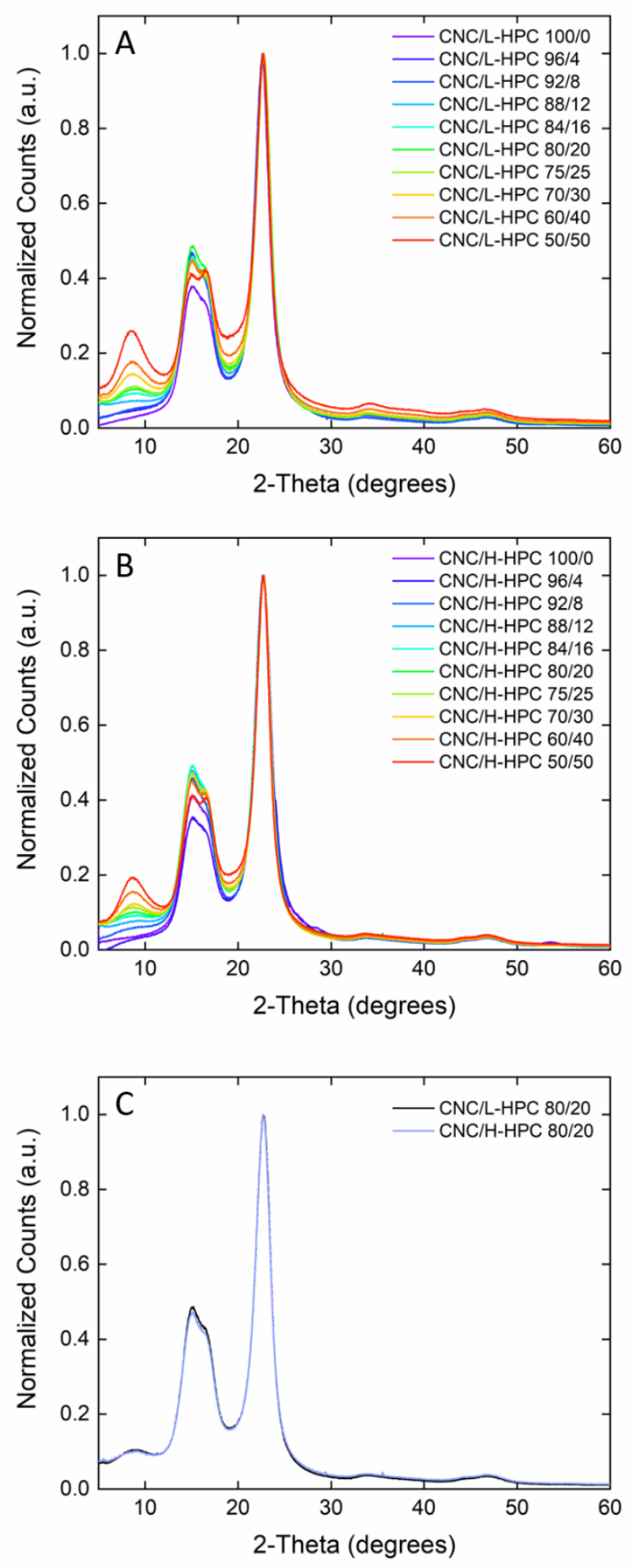

Figure S6. A-C) PXRD measurements of CNC/HPC composites. A) CNC/L-HPC composites ranging from $50 \mathrm{w} / \mathrm{w} \mathrm{CNC}$ to $100 \mathrm{w} / \mathrm{w} \mathrm{CNC}$. B) $\mathrm{CNC} / \mathrm{H}-\mathrm{HPC}$ composites ranging from $50 \mathrm{w} / \mathrm{w}$ CNC to $100 \mathrm{w} / \mathrm{w}$ CNC. C) Comparison of CNC/L-HPC and CNC/H-HPC 80/20 w/w. 

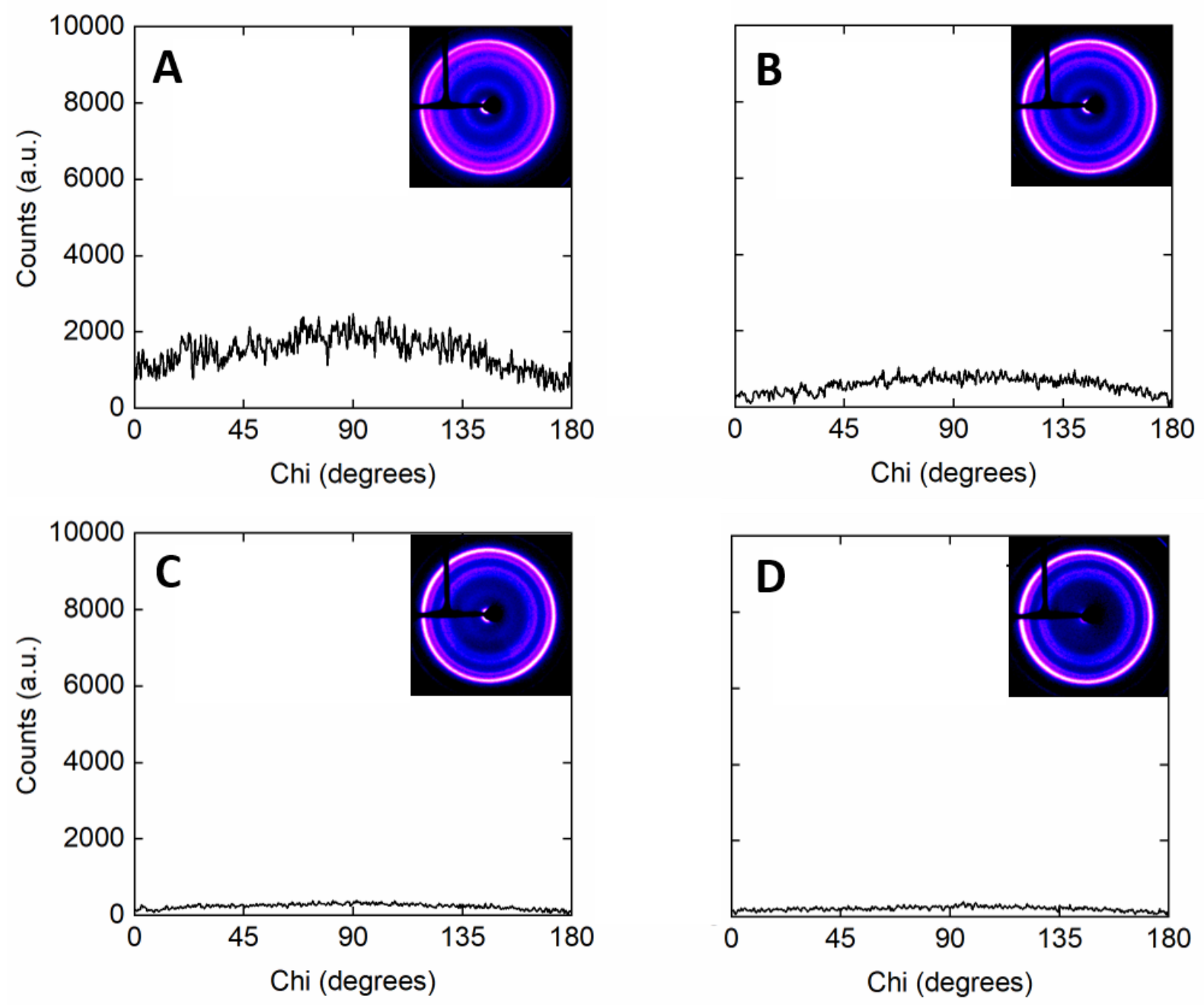

Figure S7. A-D) 2D diffraction integrated along chi at 22 degrees $2 \theta$ for A) CNC/L-HPC 50/50 w/w, B) CNC/L-HPC 60/40 w/w, C) CNC/L-HPC 80/20 w/w, and D) CNC. 

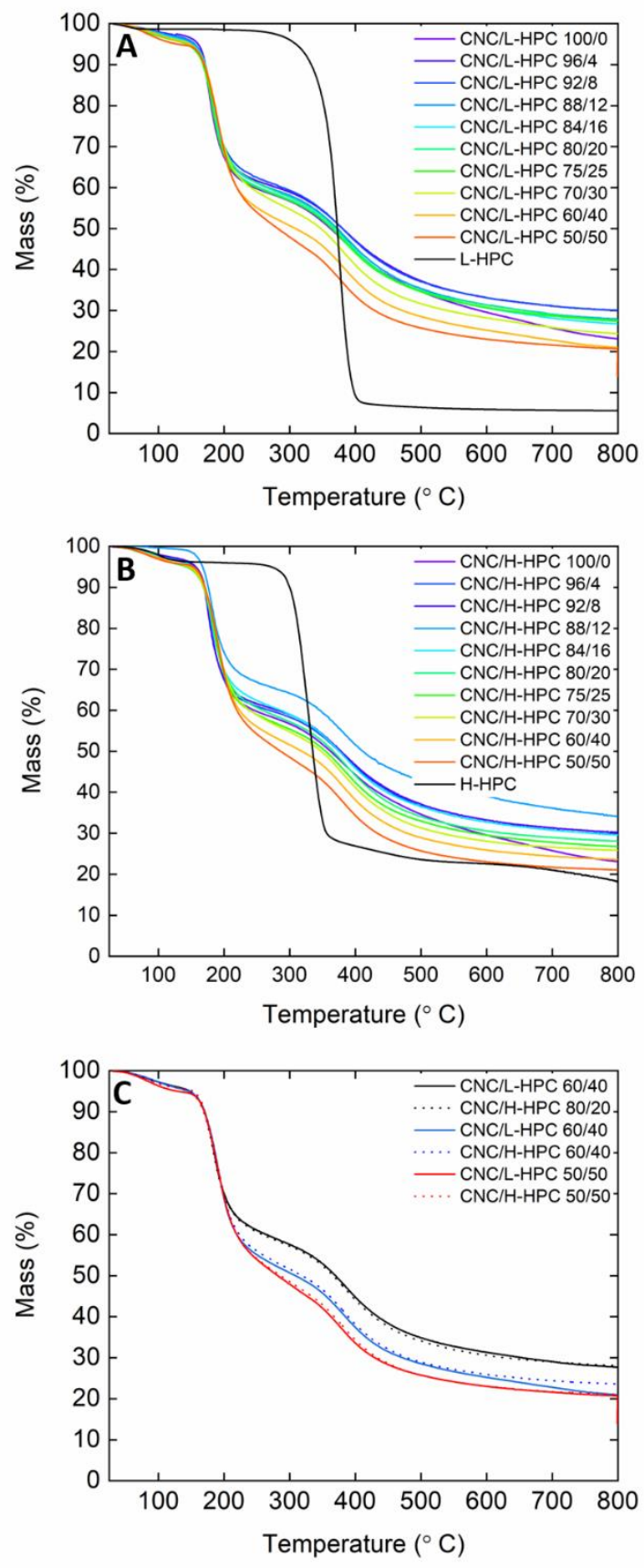

Figure S8. TGA of $\mathrm{CNC} / \mathrm{HPC}$ composites. A) $\mathrm{CNC} / \mathrm{L}-\mathrm{HPC}$ composites ranging from $50 \mathrm{w} / \mathrm{w}$ $\mathrm{CNC}$ to $100 \mathrm{w} / \mathrm{w} \mathrm{CNC}$. B) $\mathrm{CNC} / \mathrm{H}-\mathrm{HPC}$ composites ranging from $50 \mathrm{w} / \mathrm{w} \mathrm{CNC}$ to $100 \mathrm{w} / \mathrm{w} \mathrm{CNC}$. C) Comparison of CNC/L-HPC and CNC/H-HPC composite films. 

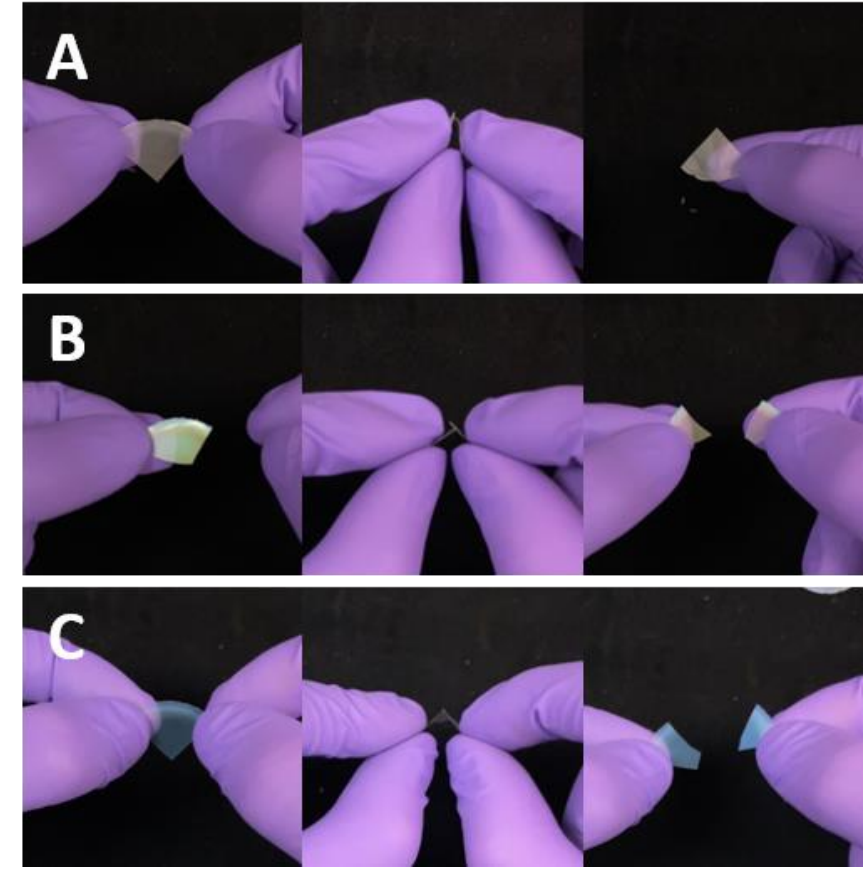

Figure S9. Photographs of CNC/H-HPC composite films before (left), during (middle), and after (right) bending. A) CNC/H-HPC 50/50 w/w, B) CNC/H-HPC 80/20 w/w, and C) CNC/H-HPC $100 / 0 \mathrm{w} / \mathrm{w}$. 


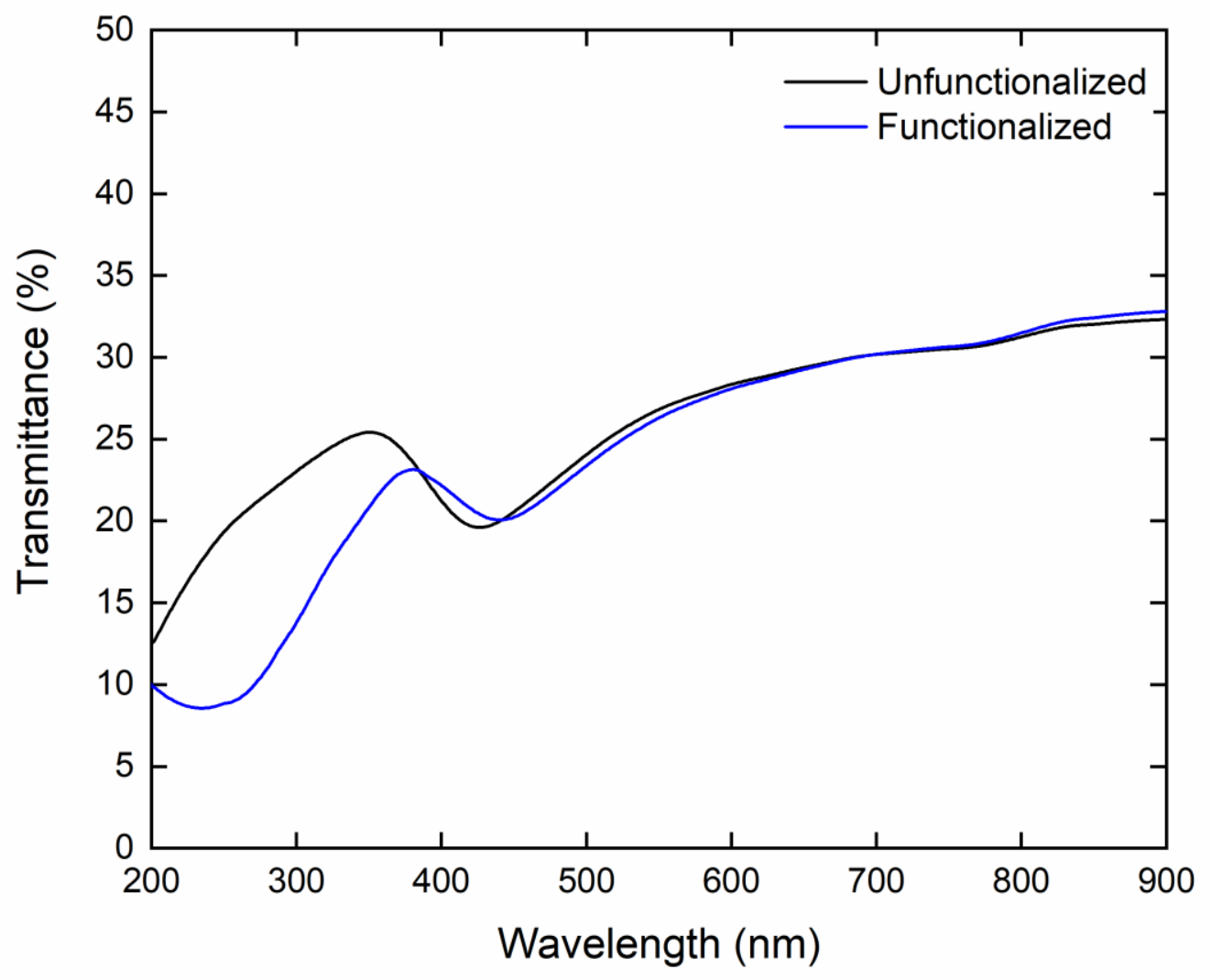

Figure S10. UV-Vis spectra of a $\mathrm{CNC} / \mathrm{HPC}$ composite film before and after treatment with methacrylic anhydride. 

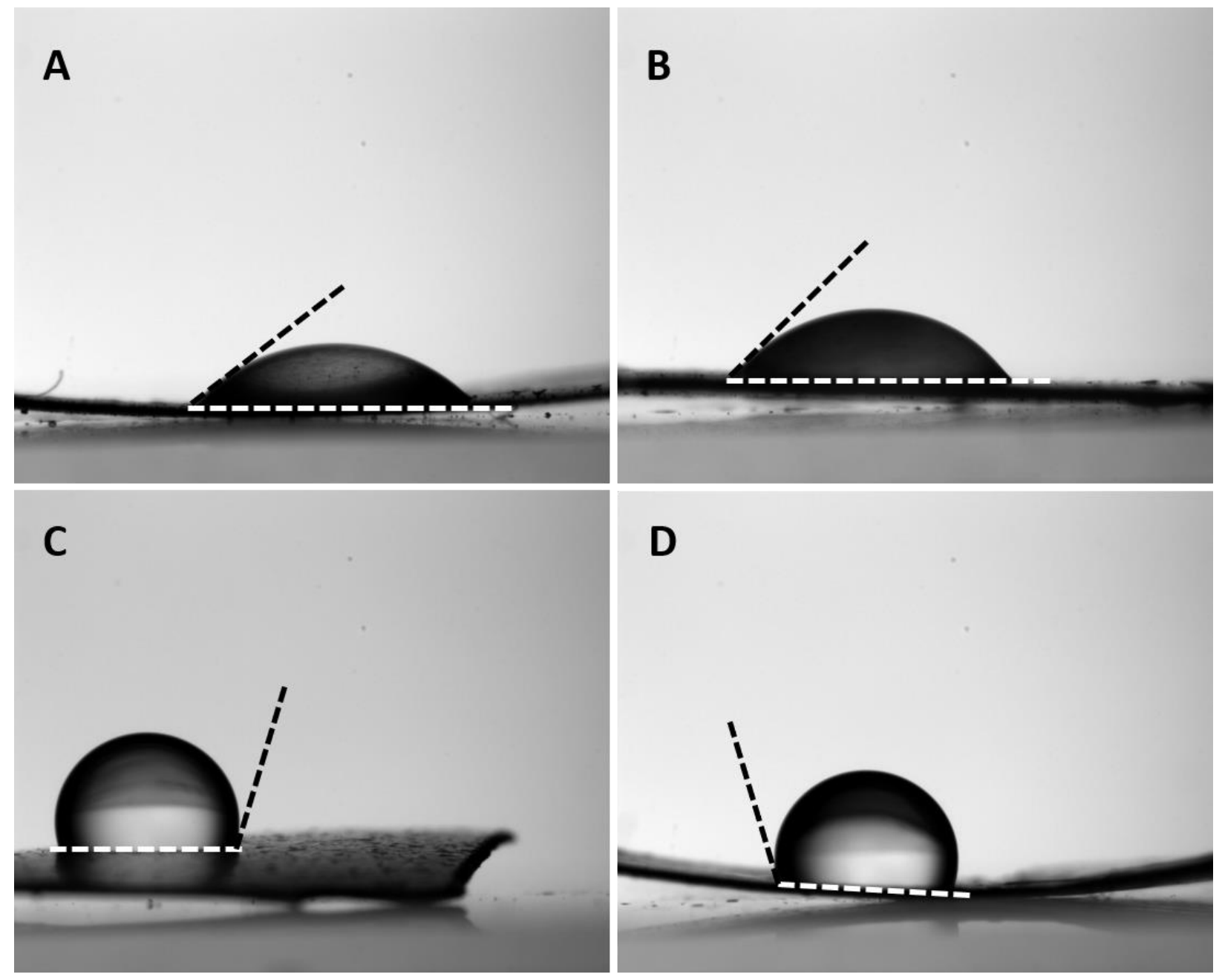

Figure S11. Water contact angle measurements for unfunctionalized A) CNC/L-HPC 60/40 w/w, and B) CNC/H-HPC 60/40 w/w, and functionalized C) CNC/L-HPC 60/40 w/w, and D) CNC/HHPC 60/40 w/w. 

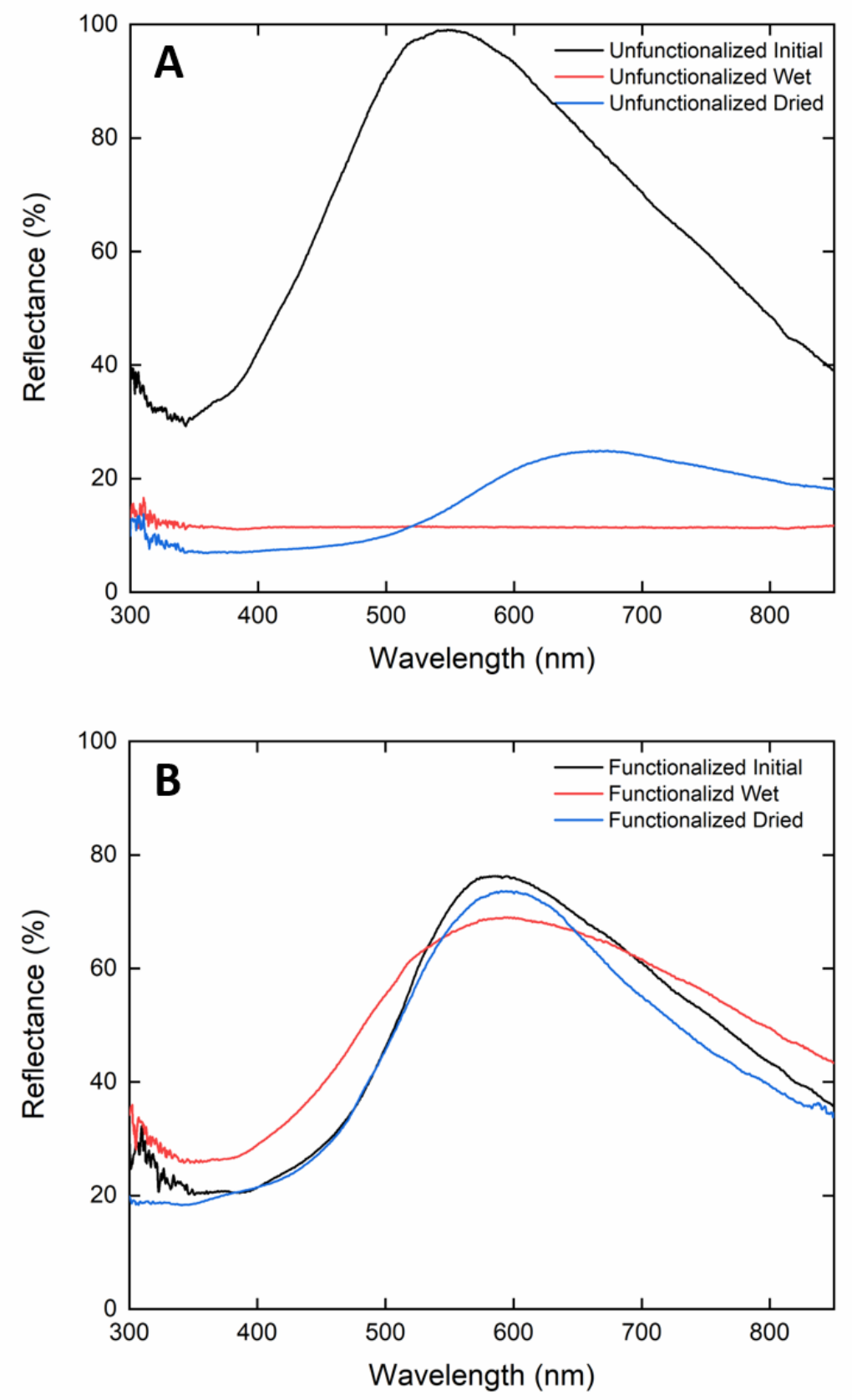

Figure S12. UV-Vis reflectance spectra of an A) unfunctionalized CNC/HPC composite film, and B) methacrylate-functionalized $\mathrm{CNC} / \mathrm{HPC}$ composite film. Reflectance measurements are of the film in its initial state, after being submerged in $80^{\circ} \mathrm{C}$ water for $15 \mathrm{~s}$, and after being allowed to dry again under ambient conditions. 


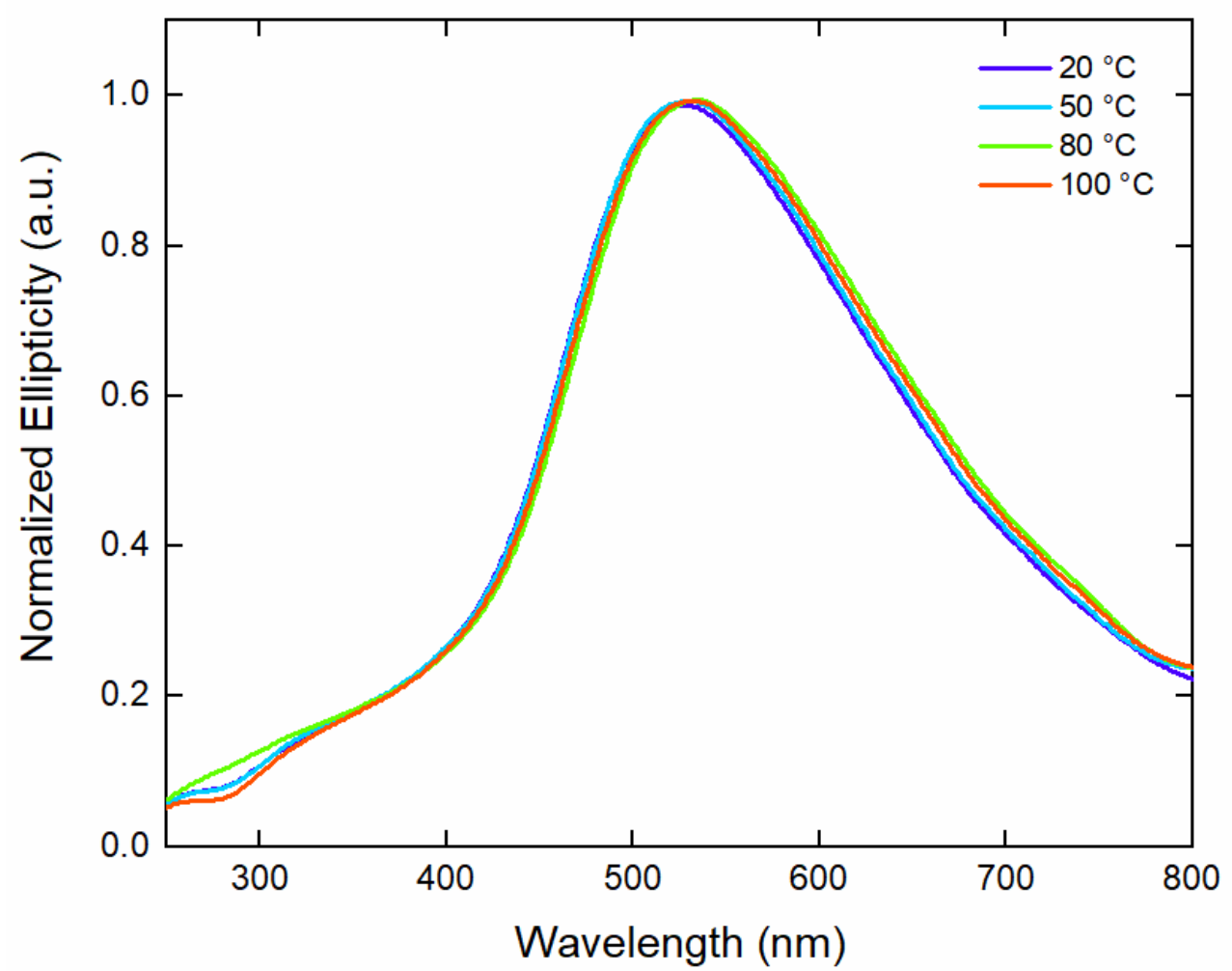

Figure S13. Variable temperature CD spectra of a dry CNC/HPC composite film. 